\title{
Highly Magnetized Accreting Pulsars: Are There Accreting Magnetars?
}

\author{
Pere Blay ${ }^{1}$, Pablo Reig ${ }^{2}$, Víctor Reglero ${ }^{1}$ \\ ${ }^{1}$ IPL, University of Valencia, Spain \\ ${ }^{2}$ University of Crete, Greece \\ Corresponding author: pere.blay@uv.es
}

\begin{abstract}
2S 0114+650, GX 301-2, IGR J16358-4726, X Per, 4U 2206+54, SXP 1062, and 3A 1954+319 are thought to possess high magnetic fields. They have recently been named accreting magnetars, or highly magnetized accreting pulsars. In this work their properties are reviewed. Within the context of their observational properties (mainly from INTEGRAL data), and the recent models of accretion onto highly magnetized neutron stars, their similarities and differences are analyzed. The aim is to find a common framework to understand the evolution (in terms of past and present history) of these sources, and to establish the basis of a possible new kind of accreting sources. Two of these sources, namely X Per and $4 \mathrm{U} 2206+54$, contain a massive main-sequence companion, while the rest are supergiant X-ray binaries or symbiotic systems. The variety of astrophysical scenarios represented by this set is wide, therefore the study of these systems is also important in order to establish commonalities between the different types of accreting X-ray pulsars and to study the possible evolutionary links between them.
\end{abstract}

Keywords: stars: binaries: close - stars: magnetars - stars: neutron - X-rays: binaries.

\section{Introduction}

Magnetars are neutron stars with bright persistent Xray emission (on the order of $\mathrm{L}_{X} \sim 10^{33-36} \mathrm{ergs}^{-1}$ ) and spin periods on the order of a few seconds. They show a very drastic spin period evolution, with large spin period derivatives and a long-term spin down tendency. The fact that they are isolated objects, together with the difficulties in considering their rotational energy as the source of the observed bright X-ray emission, are key issues in order to postulate their highlymagnetized nature (with extreme magnetic fields, on the order or greater than $\sim 10^{14}$ Gauss). Magnetars are usually grouped into two subclasses, namely Anomalous X-Ray Pulsars (AXPs) and Soft Gamma-Ray Repeaters (SGRs). For a review of magnetars see, for example, Panchapakesan (2003) and Rea \& Esposito (2011).

High Mass X-ray Binary Systems (HMXRBS) contain a massive companion $\left(\geq 10 \mathrm{M}_{\odot}\right)$ and a neutron star or a black hole. The evolution of neutron stars in HMXRBS is affected directly by the wind plasma in which they are immersed (originating from the massive companion). The ambient plasma interacts with the magnetic field of the neutron star modifying its spin period evolution. Neutron stars are born as rapid rotators. The ambient plasma removes rotational energy from the neutron star and transports it outwards and the neutron star suffers an spin-down process, which goes on until the ambient matter becomes radiative, cools rapidly, and accretion can take place. The time spent in this initial spin-down epoch will determine the final X-Ray pulsar rotational period which will be an equilibrium period which resulting from the balance of two forces: the magnetic field pressure (which stops and drags the in-falling matter), and the inward pressure exerted by the ambient plasma. Initial modelings (see, for example, Illarionov and Sunyaev, 1975) predicted that the slowest reachable pulse period of a neutron star in a binary system is on the order of $\sim 500 \mathrm{~s}$. Revisions of these initial models (see, for example, Davies and Pringle, 1981) relaxed this limit, allowing longer spin periods but only associated with higher neutron star magnetic fields.

The possible presence of magnetars in some accreting HMXRBS, in which a slowly rotating neutron star has been found, has been proposed recently by a number of authors (see, for example, Li \& van den Heuvel 1999, Finger et al. 2010, Reig et al. 2012, Ikhsanov \& Beskrovnaya 2010, Doroshenko et al. 2010, Patel et al. 2007, Popov \& Turolla 2012, Bozzo et al. 2008). Our goal is to compare the observational properties of these systems and determine which observational parameters are more suitable to derive the strength of the magnetic field. We also want to stress the difficulties in determining some of the properties of these systems when 
considering only the long-term analysis (i.e., comparing pulse periods from different epochs), or the temptation to identify spectral features as Cyclotron Resonant Scattering Features (CRSF) and derive magnetic field values from their central energy.

Despite the small number of systems, the variety of astrophysical scenarios represented by this set is wide, from main sequence accreting binary systems (X Per, SXP 1062 and 4U 2206+54) to supergiants (2S $0114+65$ and GX 301-2), and even symbiotic systems (IGR J16358-4726 and 3A 1954+319). In this sense, the study of these systems is important also to establish commonalities between the different types of HMXRBs and to study the possible evolutionary links between them. In this work we will review the observational properties of some of these systems and we will try to establish the scientific rational to develop a deeper analysis of their common properties and evolutionary status. Due to the large inhomogeneity in the energy ranges and sets of data available in the literature, we have started an analysis of these systems by using INTEGRAL/IBIS/ISGRI ${ }^{1}$ data, setting up common energy ranges, spectral resolutions, and performing a homogeneous timing analysis.

\section{The Candidates}

A number of HMXRBs have already been proposed to contain highly magnetized neutron stars, namely $2 \mathrm{~S}$ 0114+65 (Li \& van den Heuvel 1999), 4U 2206+54 (Reig et al. 2012, Finger et al. 2010), X Per (Doroshenko et al. 2012), GX 301-2 (Ikhsanov \& Finger 2012), IGR J16358-476 (Patel et al. 2007). There are also suggestions that SXP 1062 (Fu \& Li 2012) and 3A 1954+319 (Marcu et al. 2011) contain a highly magnetized neutron star. Attempts have also been made to try to explain the properties of SFXTs with the presence of a highly-magnetized neutron star with some degree of success (see Bozzo et al. 2008 ). A complete list of sources has been compiled in Table 1. Despite the fact that there are peculiarities in each of these systems which make difficult to group them as a kind (see, for example, Reig et al. 2012, Blay and Reglero 2013), we can summarize their overall commonalities:

- slowly rotating neutron stars $\left(\mathrm{P}_{\text {spin }}\right.$ slower than 500 s), with

- high magnetic fields (larger than $10^{13}$ Gauss)

- most of them show a long-term spin-down tendency
The neutron star properties in these systems are different from those of magnetars, for this reason the terms magnetar-like, or magnetar-descendant (making reference to a possible evolution from a neutron star born as a magnetar), have also been used to identify these sources (Reig et al. 2012, Blay and Reglero 2013).

Table 1: List of sources.

\begin{tabular}{lccc}
\hline Source & $\mathrm{P}_{\text {spin }}(\mathrm{s})$ & $\mathrm{P}_{\text {orb }}(\mathrm{d})$ & Type \\
\hline 4U 2206+54 & 5560 & 19.2 & MS donor \\
X Per & 864 & 250 & BeXRB \\
2S 0114+650 & 9700 & 11.6 & SXRB \\
GX 301-2 & 680 & 41.498 & SXRB \\
IGR J16358-4726 & 5880 & -- & Symbiotic \\
3A 1954+319 & 19080 & -- & Symbiotic \\
SXP 1062 & 1062 & $\sim 300$ & BeXRB \\
\hline
\end{tabular}

After 10 years of operations of the INTEGRAL satellite, a very complete and homogeneous long-term database of observations of all these sources has been compiled. We have analysed all public observations in which 4U 2206+54, X Per, 2S 0114+65, IGR J16358476, and GX 301-2 were in the field of view of INTEGRAL/IBIS/ISGRI.

The behavior of these source is similar in long time scales, with transient emission in the form of bursts or peaks and more or less continuous detection. They all show the typical behavior of wind accreting systems (although X Per is a BeX, it has been proposed that its behavior resembles that of wind-fed systems because of the large orbital distance, which will keep the neutron star away from the Be disk, see Doroshenko et al. 2012). The light curves, in every case, are complete enough to develop both short and long-term analysis of the spectral and timing properties of these systems.

The magnetic properties of the neutron stars in these systems have been investigated/explained mainly in these terms:

- spin period (neutron star evolutionary considerations)

- spectral features (Cyclotron Resonant Scattering Features, CRSF)

${ }^{1}$ INTEGRAL (INTERnational Gamma-RAy Laboratory) is an ESA mission with contributions from USA and Russia, with two high-energy imagers (IBIS, working in the energy range $15 \mathrm{kev}-10 \mathrm{MeV}$, and JEM-X sensitive in the 3-35 keV energy range) and one spectrometer (SPI, sensitive in the range $20 \mathrm{keV}-8 \mathrm{MeV}$ ). IBIS, in turn, has two detector layers, ISGRI, for the lower energy band, $15 \mathrm{keV}-1 \mathrm{MeV}$, and PiCSIT working in the $175 \mathrm{keV}-20.4 \mathrm{MeV}$ energy range. A detailed description of the mission can be found in Winkler et al. (2003) 
With regard to the spin-period history, according to Ikhsanov (2007), the maximum spin period reachable by a neutron star can be related to the intensity of its magnetic field and the quantity of accreted matter (i.e., mass accretion rate). The latter quantity is considered to be directly proportional to the X-Ray luminosity of the source (see, for example, Finger et al. 2010). The relationship between these quantities is:

$$
P_{\text {max }}=15000 \mu_{32}^{16 / 21} M_{N S}^{-4 / 21}\left(\frac{\dot{M}}{10^{15} g s^{-1}}\right)^{-5 / 7} s
$$

where $\mu_{32}$ is the dipole magnetic moment (in units of $10^{32} \mathrm{Gcm}^{-3}$ ) and $M_{N S}$ the mass of the neutron star (in units $1.5 M_{\odot}$ ), and $\dot{M}$ is the mass accretion rate. A more recent calculation of quasi-spherical accretion by Popov and Turolla 2012, yields this relationship, directly in terms of the magnetic field intensity:

$$
B_{12} \sim 8.1 \dot{M}_{16}^{1 / 3} v_{300}^{-11 / 3}\left(\frac{P_{1000}}{P_{\text {orb }, 300}}\right)^{11 / 2}
$$

where $B_{12}$ denotes the magnetic field in units of $10^{12}$ Gauss, $\dot{M}_{16}$ is the mass accretion rate in units of $10^{16} \mathrm{gs}^{-1}, v_{300}$ is the wind velocity in units of $300 \mathrm{kms}^{-1}, P_{1000}$ is the spin period in units of $1000 \mathrm{~s}$, and $P_{\text {orb }, 300}$ is the orbital period of the system in units of $300 d$.

On the other hand, a direct measurement of the magnetic field of the neutron star is possible when its high-energy spectrum shows CRSFs. CRSFs are due to the splitting of energy levels of the electron in the presence of a magnetic field. Therefore the scattering of X-ray photons by these electrons is produced at quantized Landau levels producing the absorption-like features seen in the X-Ray spectra of many HMXRBs (see Schönherr et al. 2007). The magnetic field strength can be calculated from the position of the CRSF by the formula:

$$
E_{C R S F}=11.6 \frac{B_{12}}{(1+z)}
$$

where $z$ is the gravitational redshift. It should be noted that this redshift, and consequently the measured magnetic field, will depend on the height over the neutron star surface where the CRSF is formed.

In the next sections, we will review these methods when applied mainly to some of sources listed in Table 1.

\section{Magnetic Field Determination via Spin Period Evolution}

Fig. 1 shows examples of spin period histories of $4 \mathrm{U}$ 2206+54, 2S 0114+650, IGR 16358-4726, and X Per.
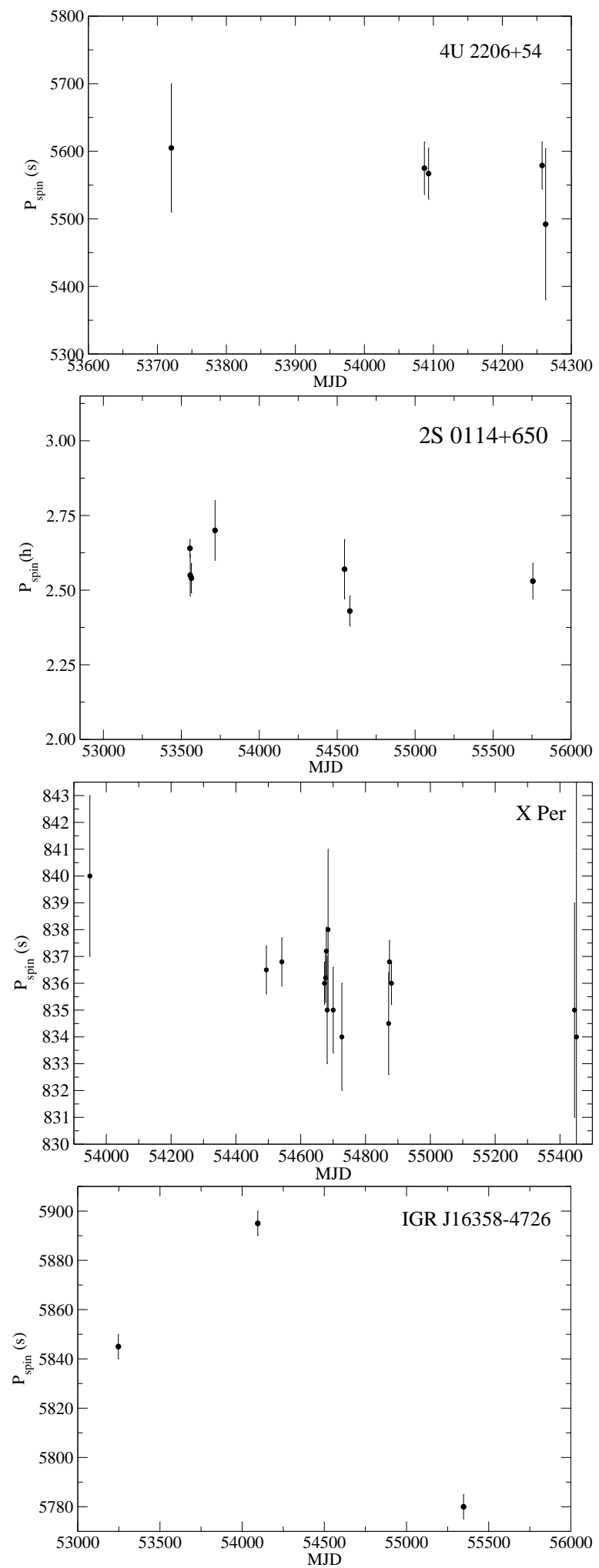

Figure 1: Spin period evolution of $4 \mathrm{U} 2206+54$ (top panel), 2S 0114+650 (second panel from the top), X Per (third panel from the top), and IGR 16358-4726 (lower panel) as obtained from INTEGRAL/IBIS/ISGRI data in the $20-40 \mathrm{keV}$ energy range. 

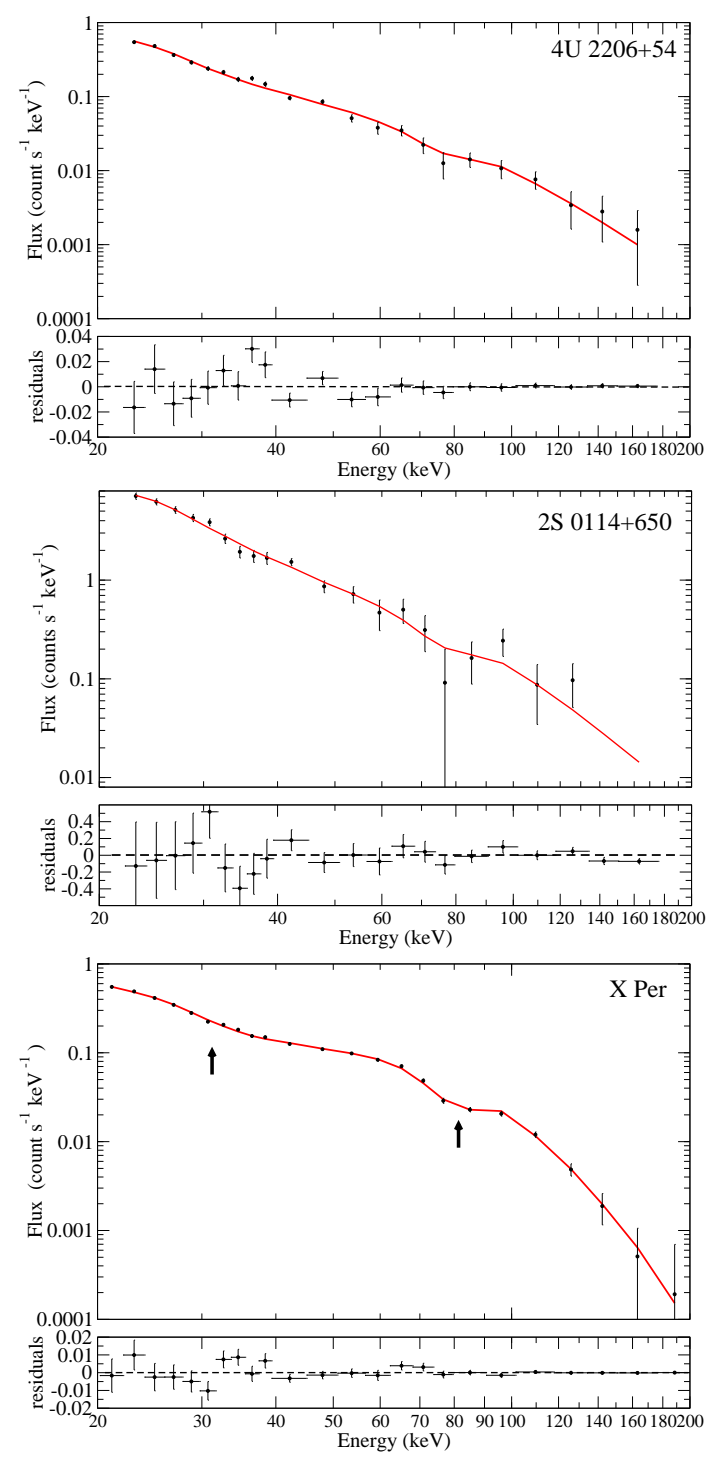

Figure 2: The 20-200 keV spectra of $4 \mathrm{U} 2206+54$ (top panel), 2S 0114+650 (middle panel) and X Per (bottom panel) extracted from INTEGRAL/IBIS/ISGRI data. The spectrum of $4 \mathrm{U} 2206+54$ has been fitted with a bulk comptonization model with a $\chi_{\text {red }}^{2} \sim 1.4$, no evidence of a feature around $\sim 30 \mathrm{keV}$ can be seen, neither in the spectrum nor in the residuals. For $2 \mathrm{~S} 0114+650$ a bulk comptonization model has been used to fit the data with a $\chi_{\text {red }}^{2} \sim 1.2$, again no evidence of a CRSF at $\sim 30 \mathrm{keV}$ is evident. For the case of $\mathrm{X}$ Per, a comptonization model and two absorption features at 31.2 $\mathrm{keV}$ and $82.3 \mathrm{keV}$ (indicated by arrows) have been used to fit the observed spectrum, with a $\chi_{\text {red }}^{2} \sim 1.1$.

In the case of $4 \mathrm{U} 2206+54$, frequency derivative values derived by Finger et al. (2010) and Reig et al. (2012) are $1.5 \times 10^{-14} \mathrm{~Hz} \mathrm{~s}^{-1}$ and $1.7 \times 10^{-14} \mathrm{~Hz} \mathrm{~s}^{-1}$ respectively. Finger et al. 2010 included an older BeppoSAX measurement. From these spin-down measure- ments, Finger et al. (2010) estimated a magnetic field of $\mathrm{B} \sim 10^{14} \mathrm{G}$. By using the relationship of Ikhsanov (2007) they also derived a magnetic field for $4 \mathrm{U} 2206+54$ on the order of $10^{14} \mathrm{G}$. Reig et al. (2012) calculated a magnetic field strength on the same order of magnitude by considering the relationship of Popov and Turolla (2012). The upper panel of Fig. 1 shows the spin period measurements from INTEGRAL/ISGRI data of $4 \mathrm{U} 2206+54$ in the period $53600-54300$ MJD. During this epoch (see Blay and Reglero 2013) the data is compatible with no changes in the spin period of the system, as the pulse period measurements show large errors. The same thing happens for X Per and 2S 0114+650 (second and third panels from the top, respectively), in which large errors in the spin period determination make the measurements compatible with no pulse period change. Therefore we cannot report on magnetic field measurements from spin period changes for these three systems.

The luminosity of $2 \mathrm{~S} 0114+65$ in the epoch shown in Fig. 1 is $4 \times 10^{37} \mathrm{ergs}^{-1}$ (estimated from the model fitted to the spectrum shown in Fig. 2), considering a distance to the source of $7.2 \mathrm{kpc}$ (Hall et al. 2000). Therefore, the Ikhsanov (2007) relationship yields a magnetic moment on the order of $10^{33} \mathrm{G} \mathrm{cm}^{-3}$, which yields a magnetic field strength of $B \sim 10^{15} \mathrm{G}$. The relationship from Popov and Turolla (2012), however, results in an unreasonable number when applied to this source.

Doroshenko et al. (2012) argue that, for the case of $\mathrm{X}$ Per, according to the torques applied to the neutron star magnetosphere in the case of wind accretion, and taking into account the theoretical approaches of Doroshenko et al. (2010), Davidson and Ostriker (1973), Davies et al. (1979), and Bisnovatyi-Kogan (1991), the magnetic field in X Per is expected to be on the order of $\mathrm{B} \sim 10^{14}$. We show in the lower panel of Fig. 1 the spin period measurements made with INTEGRAL/ISGRI data. For this source We can estimate the luminosity to be $L_{x} \sim 1.09 \times 10^{35} \mathrm{ergs}^{-1}$ in this epoch (estimated from the model fitted to the spectrum shown in Fig. 2 ). As the luminosity of the source is closely related to the accretion rate, we can estimate the field strength of X Per by using the relationship of Popov and Turolla (2012), yielding $B \sim 10^{12} \mathrm{G}$, which falls within the range of normal neutron star magnetic fields. The relationship from Ikhsanov (2007) results in a magnetic moment of $\mu \sim 10^{30} \mathrm{G} \mathrm{cm}^{-3}$, which implies a magnetic field on the order of $B \sim 10^{12} \mathrm{G}$. We find, therefore, a discrepancy with the results of Doroshenko et al. (2012). We cannot reproduce their calculations, however, because of the large errors in the spin period determination, which prevent the determination of a reliable frequency derivative, needed for the determination of the spin torques.

For IGR J16358-4726 we see in the lower panel of 
Fig. 1 that the source experiences large pulse period changes. It shows a spin-down between MJD 53250 and MJD 54000 of $\dot{\nu} \sim 2.4 \times 10^{-14} \mathrm{~Hz} \mathrm{~s}^{-1}$. According to the Equation (9) from Finger et al. (2010), which relates the magnetic moment of the neutron star with the measured spin-down, we find in IGR J16358-4726 a minimum magnetic moment of $\mu \sim 2 \times 10^{32} \mathrm{G} \mathrm{cm}^{-3}$, which implies a magnetic field on the order of or larger than $\mathrm{B} \sim 10^{14} \mathrm{G}$.

These calculations rely on a good measurement of the luminosity (or mass accretion rate) or of the changes in the spin period. The major uncertainty comes from the determination of the luminosity of the source, calculated from the source flux in a given energy range and the estimated distance to the source, which is usually subject to large uncertainties. With respect to the use of spin period changes, the estimates always rely on long-term or average behavior. On the one hand this is due to the poor knowledge of the short-term or detailed pulse period evolution in many cases, and, on the other hand, to the lack of a detailed and complete theoretical description of the wind accretion scenario. The efforts of Postnov et al. (2013), however, try to fill this lack of good theoretical approximations. We will comment on that in the last section.

\section{Magnetic Field Determination via Cyclotron Resonant Scatering Features}

A more direct measurement of the magnetic field strength of the neutron star in these systems can be obtained by determining the position of the fundamental CRSF seen in their X-Ray spectra. CRSF have been reported in GX 301-02 (La Barbera et al. 2005, Makishima and Mihara 1992), 2S 0114+65 (Wang 2010, Bonning and Falanga 2005), 4U 2206+54 (Blay et al. 2005, , Wang 2009, Torrejón et al. 2004, Masseti et al. 2004), and X Per (this work, see Fig. 2, Doroshenko et al. 2010), see Table 2.

Table 2 summarizes the CRSF reported in these systems and the magnetic field strength measurement derived.

Table 2: CRSFs and B determination. See references in the text.

\begin{tabular}{lcc}
\hline Source & $\mathrm{E}_{C R S F}(\mathrm{keV})$ & $\mathrm{B}\left(10^{12}\right.$ Gauss $)$ \\
\hline $4 \mathrm{U} 2206+54$ & 30 & 2.6 \\
X Per & 31.2 & 2.7 \\
2S 0114+650 & 22 & 1.9 \\
GX 301-2 & 37 & 3.2 \\
5 2S 0114+650 & $22^{(c)}$ & 1.9 \\
\hline
\end{tabular}

We see in all cases that the magnetic field strength derived by this method is on the order of those found in other neutron stars in HMXRBs, i.e., 100 to 1000 times lower than those of the magnetars. We find, therefore, a discrepancy in magnetic field strength determination with respect to the methods shown in the previous section. One could think that the magnetic fields derived from spin period evolution are not properly calculated, in view of the more direct determination from CRSF measurements. However the location of CRSF is not free of ambiguities. In the case of $4 \mathrm{U} 2206+54$ (see top panel of Fig. 2), for example, this feature has been observed only marginally, and only at one epoch (see Blay et al. 2005 and references there in). It has not been observed again (see Blay and Reglero 2013). Therefore, although it has been assumed that the absorption observed was a CRSF, this may not be the case. A similar situation applies to $2 \mathrm{~S} 0114+65$ (see middle panel of Fig. 2), in which the detection of the possible CRSF has only been marginal (see Wang 2010) and not confirmed again. In the case of X Per, Doroshenko et al. (2010) show how instead of a CRSF fundamental and its first harmonic (see bottom panel of Fig. 2), the X Per spectra can be very well described by a combination of two comptonization models (compTT + compTT, in XSPEC notation) demonstrating the possibility that the features observed may not be CRSFs.

Therefore, at least in some cases, the possibility of finding magnetar-like magnetic fields in these systems is not ruled out by the reported observations of CRSFs.

\section{Discussion and Conclusion}

We have seen that in most cases the determination of the magnetic field strength of the neutron star is ambiguous, or even contradictory, when the result from different approaches are compared. We want to emphasize how different approaches may seem to result in different magnetic field strength estimates. An homogeneous and coherent approach is needed in order to determine if there are links between these systems (in terms of their spin period and binary evolution) and to support or discard the different theories trying to explain the puzzling presence of long spin periods in HMXRBs.

There have been recent theoretical efforts in order to provide better modeling of the wind-accretion scenario. Ikshanov and Beskrovnaya (2013), for example, report on a likely explanation of the large spin-down shown in $4 \mathrm{U} 2206+54$ by taking into account a higher than usual magnetic field of the companion star and, consequently, that the accreting material can be magnetized. The possibility of an optical companion with a large magnetic field had been already proposed by Blay and Reglero (2011). 
The efforts of Postnov et al. (2013) in modeling more accurately the wind accretion mechanism in HMXRBs also result in magnetic field estimations for $4 \mathrm{U} 2206+54$ and SXP 1062 on the order of typical magnetic fields found in accreting X-Ray pulsars $\left(\sim 10^{12} G\right)$. They explain the behavior of sources like GX 301-2 with the hypothesis of being older systems which have already reached their equilibrium period.

Together with an improvement in the modeling of wind accretion in HMXRBs, a better observational approach is needed in order to understand the behavior of these sources. Multiwavelength campaigns (IR, optical, UV, X-rays) on these objects are needed (as simultaneous as possible) in order to determine the various parameters involved: obtain more accurate spectral classification and orbital solutions, measure accurate mass loss rates and wind velocity laws, etc.

\section{Acknowledgement}

P. Blay acknowledges funding from the Spanish Ministerio de Economia y Competitividad through project AYA-2011-29936-C05.

\section{References}

[1] Bisnovatyi-Kogan, G. S. 1991, A\&A, 245, 528

[2] Blay P., Ribó M., Negueruela I., Torrejn J. M., Reig P., Camero A., Mirabel, I.F., \& Reglero V. 2005, A\&A 438, 936

[3] Blay P. and Reglero V. 2011, BSRSL, 80, 634

[4] Blay P. and Reglero V. 2013, PoS(INTEGRAL 2012)012

[5] Bonning E. W., Falanga M. 2005, A\&A, 436, L31

[6] Bozzo E., Falanga M., Stella L. 2008, ApJ, 683, 1031 doi:10.1086/589990

[7] Davidson, K., and Ostriker, J. P. 1973, ApJ, 179, 585

[8] Davies, R. E., Fabian, A. C., and Pringle, J. E. 1979, MNRAS, 186, 779

[9] Davies R.E., and Pringle J.E. 1981, MNRAS, 196, 209

[10] Doroshenko V., Santangelo A., Suleimanov V., Kreykenbohm I., Staubert R., Ferrigno C., Klochkov D. 2010, A\&A 515, A10

[11] Doroshenko V., Santangelo A., Kreykenbohm I., and Doroshenko R. 2012, A\&A 540, L1
[12] Finger M.H., Ikhsanov N.R., Wilson-Hodge C.A., \& Patel S.K. 2010., ApJ, 709, 1249 doi:10.1088/0004-637X/709/2/1249

[13] Fu L., Li X.D. 2012, ApJ, 757,171 doi:10.1088/0004-637X/757/2/171

[14] Fürst F., Marcu D.M., Pottschmidt K., Grinberg V., Wilms J., and Cadolle Bel M. 2011, PoS(INTEGRALL 2010)017

[15] Hall T. A., Finley J. P., Corbet R. H. D., Thomas R. C., 2000, ApJ, 536, 450 doi:10.1086/308924

[16] Harbel, F., Sturm, R., \& Filipović, M. D. et al. 2012, A\&A, 537, L1

[17] Ikhsanov, N. R. 2007, MNRAS, 375, 698 doi:10.1111/j.1365-2966.2006.11331.x

[18] Ikhsanov N.R., Finger M.H. 2012, ApJ, 753,1 doi:10.1088/0004-637X/753/1/1

[19] Ikhsanov N.R., Beskrovnaya N.G. 2010, Astrophysics, 53-2, 237

[20] Ikhsanov N. R. and Beskrovnaya N. G.2013, ARep, 57,287

[21] Illarionov A.F., and Sunyaev R.A. 1975, A\&A, 39,185

[22] Koh D.T., Bildsten L., Chakrabarty D., et al. 1997, ApJ, 479, 933

[23] Li X. D., van den Heuvel E. P. J., 1999, ApJ, 513, L45

[24] La Barbera, A., Segreto, A., Santangelo, A., Kreykenbohm, I., \& Orlandini, M. 2005, A\&A, 438, 617

[25] Makishima K., Mihara T. 1992, in Magnetic Fields of Neutron Stars, ed. T. Tanaka \& K. Koyama (Tokyo: Universal Academy Press), 23

[26] Marcu D. M., Fürst F., Pottschmidt K., et al. 2011, ApJ, 742, L11 doi:10.1088/2041-8205/742/1/L11

[27] Masetti N., Dal Fiume D., Amati L., Del Sordo S., Frontera F., Orlandini M., and Palazzi E. 2004, A\&A, 423, 311

[28] Panchapakesan N. 2003, Bull. Astr. Soc. India, 31, 19

[29] Patel S.K., Zurita J., Del Santo M. et al. 2007, ApJ, 657, 994 doi:10.1086/510374

[30] Popov S.B., Turolla R. 2012, MNRAS, 421, L127 doi:10.1111/j.1745-3933.2012.01220.x 
[31] Postnov K.A., Shakura N.I., Kochetkova A.Yu., and Hjalmarsdotter L., 2013, arXiv:1307.3032

[32] Rea N., Esposito P. 2011, in High-Energy Emission from Pulsars and their Systems, ed. D. F. Torres \& N. Rea, Astrophysics and Space Science Proceedings (Springer Berl in Heidelberg), 247

[33] Reig P., Torrejón J.M., \& Blay P. 2012, MNRAS, 425, 595 doi:10.1111/j.1365-2966.2012.21509.x

[34] Schönherr G., Wilms J., Kretschmar P., Kreykenbohm I., Santangelo A., Rothschild R.E., Coburn
W., Staubert R. 2007, A\&A 472, 353

[35] Torrejn J. M., Kreykenbohm I., Orr A., Titarchuk L., and Negueruela I. 2004, A\&A, 423, 301

[36] Wang, W. 2009, MNRAS, 398, 1428 doi:10.1111/j.1365-2966.2009.15200.x

[37] Wang W. 2010, A\&A 516, A15

[38] Winkler C., Courvoisier T.J.L., Di Cocco G., et al. 2003, A\&A, 411, L1 